PROCEEDINGS OF THE

AMERICAN MATHEMATICAL SOCIETY

Volume 132, Number 3, Pages 641-647

S 0002-9939(03)07147-8

Article electronically published on September 29, 2003

\title{
A NEGATIVE ANSWER TO NEVANLINNA'S TYPE QUESTION AND A PARABOLIC SURFACE WITH A LOT OF NEGATIVE CURVATURE
}

\author{
ITAI BENJAMINI, SERGEI MERENKOV, AND ODED SCHRAMM \\ (Communicated by Jozef Dodziuk) \\ In memory of Bob Brooks
}

\begin{abstract}
Consider a simply-connected Riemann surface represented by a Speiser graph. Nevanlinna asked if the type of the surface is determined by the mean excess of the graph: whether mean excess zero implies that the surface is parabolic, and negative mean excess implies that the surface is hyperbolic. Teichmüller gave an example of a hyperbolic simply-connected Riemann surface whose mean excess is zero, disproving the first of these implications. We give an example of a simply-connected parabolic Riemann surface with negative mean excess, thus disproving the other part. We also construct an example of a complete, simply-connected, parabolic surface with nowhere positive curvature such that the integral of curvature in any disk about a fixed basepoint is less than $-\epsilon$ times the area of disk, where $\epsilon>0$ is some constant.
\end{abstract}

\section{INTRODUCTION}

The uniformization theorem states that for every simply-connected Riemann surface $X$ there exists a conformal map $\varphi: X_{0} \rightarrow X$, where $X_{0}$ is either the complex plane $\mathbb{C}$, the open unit disc $\mathbb{D}=\{z \in \mathbb{C}:|z|<1\}$, or the extended complex plane (Riemann sphere) $\overline{\mathbb{C}}=\mathbb{C} \cup\{\infty\}$, and these possibilities are mutually exclusive [1]. The map $\varphi$ is called the uniformizing map. A simply-connected Riemann surface $X$ is said to have hyperbolic, parabolic, or elliptic type, according to whether it is conformally equivalent to $\mathbb{D}, \mathbb{C}$, or $\overline{\mathbb{C}}$, respectively. In what follows, we assume that $X$ is not compact, thus excluding the elliptic case from consideration.

We are interested in the application of the Uniformization Theorem to the following construction. A surface spread over the sphere is a pair $(X, \psi)$, where $X$ is a topological surface and $\psi: X \rightarrow \overline{\mathbb{C}}$ a continuous, open and discrete map. The map $\psi$ is called a projection. Two such surfaces $\left(X_{1}, \psi_{1}\right),\left(X_{2}, \psi_{2}\right)$ are equivalent, if there exists a homeomorphism $\phi: X_{1} \rightarrow X_{2}$, such that $\psi_{1}=\psi_{2} \circ \phi$. According to a theorem of Stoilow [10], a continuous open and discrete map $\psi$ near each point $z_{0}$ is homeomorphically equivalent to a map $z \mapsto z^{k}$. The number $k=k\left(z_{0}\right)$ is called the local degree of $\psi$ at $z_{0}$. If $k \neq 1, z_{0}$ is called a critical point and $\psi\left(z_{0}\right)$ a critical value. The set of critical points is a discrete subset of $X$. The theorem

Received by the editors October 17, 2002.

2000 Mathematics Subject Classification. Primary 14J15, 60 J65.

The research of the second author was supported by NSF grant DMS-0072197.

(C)2003 American Mathematical Society 
of Stoïlow implies that there exists a unique conformal structure on $X$ that makes $\psi$ into a meromorphic function. If $X$ is simply-connected, what is the type of the Riemann surface so obtained? This is one version of the type problem. Equivalent surfaces have the same type.

Rolf Nevanlinna's problem concerns a particular class of surfaces spread over the sphere, denoted by $F_{q}$. Let $\left\{a_{1}, \ldots, a_{q}\right\}$ be distinct points in $\overline{\mathbb{C}}$.

Definition. A surface $(X, \psi)$ belongs to the class $F_{q}=F\left(a_{1}, \ldots, a_{q}\right)$ if $\psi$ restricted to the complement of $\psi^{-1}\left(\left\{a_{1}, \ldots, a_{q}\right\}\right)$ is a covering map onto its image $\overline{\mathbb{C}} \backslash\left\{a_{1}, \ldots, a_{q}\right\}$.

Assume that $(X, \psi) \in F_{q}$ and $X$ is noncompact. We fix a Jordan curve $L$, visiting the points $a_{1}, \ldots, a_{q}$ in cyclic order. The curve $L$ is usually called a base curve. It decomposes the sphere into two simply-connected regions $H_{1}$, the region to the left of $L$, and $H_{2}$, the region to the right of $L$. Let $L_{i}, i=1,2, \ldots, q$, be the arc of $L$ from $a_{i}$ to $a_{i+1}$ (with indices taken modulo $q$ ). Let us fix points $p_{1}$ in $H_{1}$ and $p_{2}$ in $H_{2}$, and choose $q$ Jordan $\operatorname{arcs} \gamma_{1}, \ldots, \gamma_{q}$ in $\overline{\mathbb{C}}$, such that each arc $\gamma_{i}$ has $p_{1}$ and $p_{2}$ as its endpoints, and has a unique point of intersection with $L$, which is in $L_{i}$. We take these arcs to be interiorwise disjoint, that is, $\gamma_{i} \cap \gamma_{j}=\left\{p_{1}, p_{2}\right\}$ when $i \neq j$. Let $\Gamma^{\prime}$ denote the graph embedded in $\overline{\mathbb{C}}$, whose vertices are $p_{1}, p_{2}$, and whose edges are $\gamma_{i}, i=1, \ldots, q$, and let $\Gamma=\psi^{-1}\left(\Gamma^{\prime}\right)$. We identify $\Gamma$ with its image in $\mathbb{R}^{2}$ under an orientation-preserving homeomorphism of $X$ onto $\mathbb{R}^{2}$. The graph $\Gamma$ has the following properties:

(1) $\Gamma$ is infinite, connected;

(2) $\Gamma$ is homogeneous of degree $q$;

(3) $\Gamma$ is bipartite.

A graph, properly embedded in the plane and satisfying these properties is called a Speiser graph, also known as a line complex. The vertices of a Speiser graph $\Gamma$ are traditionally marked by $\times$ and $\circ$, such that each edge of $\Gamma$ connects a vertex marked $\times$ with a vertex marked $\circ$. Such a marking exists, since $\Gamma$ is bipartite. Each face of $\Gamma$, i.e., connected component of $\mathbb{R}^{2} \backslash \Gamma$, has either a finite even number of edges along its boundary, in which case it is called an algebraic elementary region, or infinitely many edges, in which case it is called a logarithmic elementary region. Two Speiser graphs $\Gamma_{1}, \Gamma_{2}$ are said to be equivalent, if there is a sense-preserving homeomorphism of the plane that takes $\Gamma_{1}$ to $\Gamma_{2}$.

The above construction is reversible. Suppose that the faces of a Speiser graph $\Gamma$ are labelled by $a_{1}, \ldots, a_{q}$, so that when going counterclockwise around a vertex $x$, the indices are encountered in their cyclic order, and around $\circ$ in the reversed cyclic order. We fix a simple closed curve $L \subset \overline{\mathbb{C}}$ passing through $a_{1}, \ldots, a_{q}$. Let $H_{1}, H_{2}, L_{1}, \ldots, L_{q}$ be as before. Let $\Gamma^{*}$ be the planar dual of $\Gamma$. If $e$ is an edge of $\Gamma^{*}$ from a face of $\Gamma$ marked $a_{j}$ to a face of $\Gamma$ marked $a_{j+1}$, let $\psi$ map $e$ homeomorphically onto the corresponding $\operatorname{arc} L_{j}$ of $L$. This defines $\psi$ on the edges and vertices of $\Gamma^{*}$. We then extend $\psi$ to the faces of $\Gamma^{*}$ in the obvious way. This defines a surface spread over the sphere $\left(\mathbb{R}^{2}, \psi\right) \in F\left(a_{1}, \ldots, a_{q}\right)$. See [6] for further details.

For a Speiser graph $\Gamma$, Nevanlinna introduces the following characteristics. Let $V(\Gamma)$ denote the set of vertices of the graph $\Gamma$. To each vertex $v \in V(\Gamma)$ we assign the number

$$
E(v)=2-\sum_{f \in F(v)}\left(1-1 / k_{f}\right)
$$


where $F(v)$ denotes the set of faces containing $v$ on their boundary and $2 k_{f}$ is the number of edges on the boundary of $f, k_{f} \in\{1,2, \ldots, \infty\}$. The function $E: V(\Gamma) \rightarrow \mathbb{R}$ is called the excess. This definition is motivated by considering the curvature, as follows. The $\psi$-pullback of the spherical metric $2|d w| /\left(1+|w|^{2}\right)$ is generally singular, i.e., it may degenerate on $\psi^{-1}\left(\left\{a_{1}, \ldots, a_{q}\right\}\right)$. The surface $X$, endowed with the pullback metric, is a spherical polyhedral surface, which is a particular kind of orbifold. The integral curvature $\omega$ on $X$ is a signed Borel measure, so that for each Borel subset $B \subset X, \omega(B)$ is the area of $B$ with respect to the pullback metric minus $2 \pi \sum_{z}\left(k_{z}-1\right)$, where the sum is over all critical points $z \in B$ and $k_{z}$ is the local degree of $\psi$ at $z$.

Each vertex of $\Gamma$ represents a hemisphere, and each face of $\Gamma$ with $2 k$ edges represents a critical point, where $k$ is the local degree of $f$ at this point. Therefore, each vertex of $\Gamma$ has positive integral curvature $2 \pi$, and each face with $2 k$ edges has negative integral curvature $-2 \pi(k-1)$. We assign the negative curvature evenly to all the vertices of the face. A face with infinitely many edges contributes $-2 \pi$ to each vertex on its boundary. The curvature assigned to every $v \in V(\Gamma)$ is exactly $2 \pi E(v)$.

Nevanlinna also defines the mean excess of a Speiser graph $\Gamma$. We fix a base vertex $v_{0} \in V(\Gamma)$, and consider an exhaustion of $\Gamma$ by a sequence of finite graphs $\Gamma_{(i)}$, where $\Gamma_{(i)}$ is the ball of combinatorial radius $i$, centered at $v$. By averaging $E$ over all the vertices of $\Gamma_{(i)}$, and taking the limit, we obtain the mean excess, denoted $E_{m}=E_{m}(\Gamma)$, provided that the limit exists. If the limit does not exist, we consider the upper mean excess $\bar{E}_{m}$ and lower mean excess $\underline{E}_{m}$, which are the upper (lim sup) and lower (lim inf) limits, respectively.

Nevanlinna's Problem [5]. Does $\underline{E}_{m} \geq 0$ imply that the surface $X$ with the pullback complex structure is parabolic? Conversely, does $\bar{E}_{m}<0$ imply that it is hyperbolic?

Teichmüller [11 constructed an example of a surface with hyperbolic type, for which the mean excess is zero, thus giving a negative answer to the first question.

We will shortly prove that the answer to the other question is negative as well, by constructing a parabolic surface $\left(\mathbb{R}^{2}, \psi\right) \in F_{3}$ with $E_{m}<0$.

In Section 3, we shall construct an example of a nonpositively curved, simplyconnected, complete, parabolic surface, whose curvature in any ball about a fixed basepoint is less than a negative constant times the area of the ball.

\section{Counterexample}

P. Doyle 3 proved that the surface $(X, \psi)$ is parabolic if and only if a certain modification of the Speiser graph is recurrent. (See [4] and [9] for background on recurrence and transience of infinite graphs.) In the particular case where $k_{f}$ is bounded, the recurrence of the Speiser graph itself is equivalent to $(X, \psi)$ being parabolic. Though we will not really need this fact, it is not too hard to see that in a Speiser graph satisfying $E_{m}<0$ the number of vertices in a ball grows exponentially with the radius. Thus, we may begin searching for a counterexample by considering recurrent graphs with exponential growth. A very simple standard example of this sort is a tree constructed as follows. In an infinite 3-regular tree $T_{3}$, let $v_{0}, v_{1}, \ldots$ be an infinite simple path. Let $T$ be the set of vertices $u$ in $T_{3}$ such that $d\left(u, v_{n}\right) \leq n$ for all sufficiently large $n$. Note that there is a unique infinite 


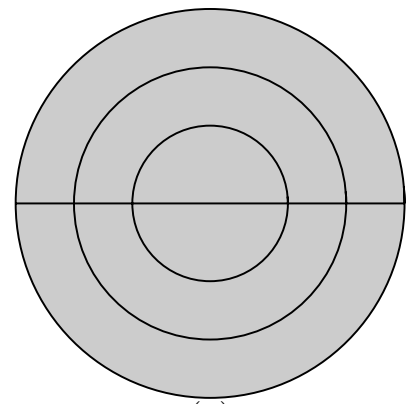

(a)

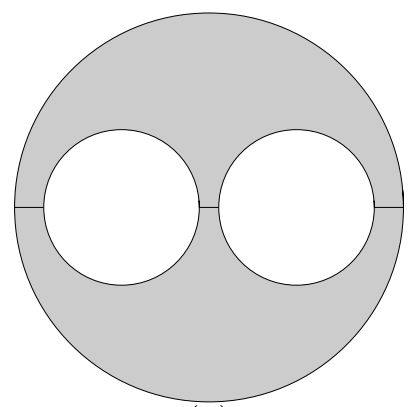

(b)

Figure 1. The surfaces $S(v)$. (a) shows $S(v)$ for a leaf $v$, where $s=2$. (b) shows $S(v)$ for a degree 3 vertex.

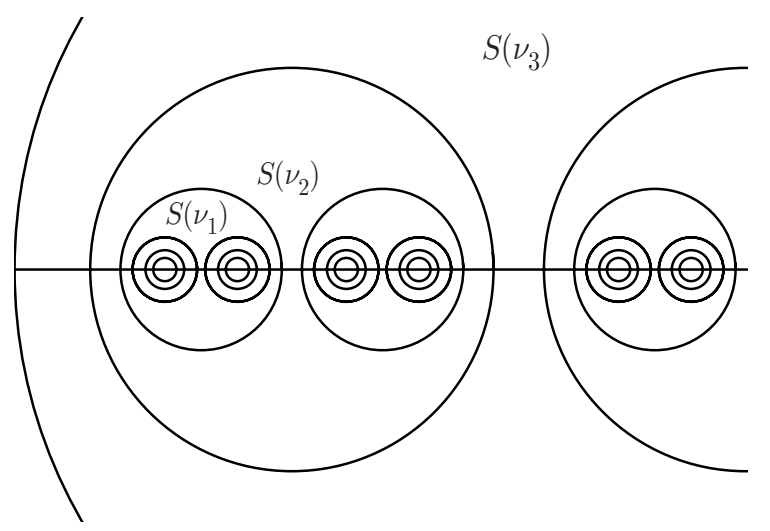

Figure 2. The Speiser graph with $s=2$. The disk $S\left(v_{0}\right)$ is the left "eye" in $S\left(v_{1}\right)$.

simple path in $T$ starting from any vertex $u$. This implies that $T$ is recurrent. It is straightforward to check that the number of vertices of $T$ in the ball $B\left(v_{0}, r\right)$ grows exponentially with $r$.

Our Speiser graph counterexample is a simple construction based on the tree $T$. Fix a parameter $s \in\{1,2, \ldots\}$, whose choice will be discussed later. To every leaf (degree-one vertex) $v$ of $T$ associate a closed disk $S(v)$ and on it draw the graph indicated in Figure 1(a), where the number of concentric circles, excluding $\partial S(v)$, is $s$. If $v$ is not a leaf, then it has degree 3 . We then associate to it the graph indicated in Figure 1(b), drawn on a triply connected domain $S(v)$. We combine these to form the Speiser graph $\Gamma$ as indicated Figure 2 by pasting the outer boundary of the surface corresponding to each vertex into the appropriate inner boundary component of its parent. Here, the parent of $v$ is the vertex $v^{\prime} \operatorname{such}$ that $d\left(v^{\prime}, v_{n}\right)=d\left(v, v_{n}\right)-1$ for all sufficiently large $n$.

Every vertex of $\Gamma$ has degree 4 and every face has 2, 4 or 6 edges on its boundary. Therefore, $\Gamma$ is a Speiser graph. Consequently, as discussed above, there is a surface spread over the sphere $X=\left(\mathbb{R}^{2}, \psi\right)$ whose Speiser graph is $\Gamma$. It is immediate to verify that $\Gamma$ is recurrent, for example, by the Nash-Williams criterion. Doyle's Theorem [3] then implies that $X$ is parabolic. Alternatively, one can arrive at the 
same conclusion by noting that there is an infinite sequence of disjoint isomorphic annuli on $\left(\mathbb{R}^{2}, \Gamma\right)$ separating any fixed point from $\infty$, and applying extremal length. (See [1], [8] for the basic properties of extremal length.)

We now show that $\bar{E}_{m}<0$ for $\Gamma$. Note that the excess is positive only on vertices on the boundary of 2-gons, which arise from leaves in $T$. On the other hand, every vertex of degree 3 in $T$ gives rise to vertices in $\Gamma$ with negative excess. Take as a basepoint for $\Gamma$ a vertex $w_{0} \in S\left(v_{1}\right)$ with negative excess, say. It is easy to see that there are constants, $a>1, c>0$ such that the number $n_{r}^{-}$of negative excess vertices in the combinatorial ball $B\left(w_{0}, r\right)$ about $w_{0}$ satisfies $c a^{r} \leq n_{r}^{-} \leq a^{r} / c$.

If $w$ is a vertex with positive excess, then there is a unique vertex $\sigma(w)$ with negative excess closest to $v$; in fact, if $w \in S(v)$, then $\sigma(w)$ is the closest vertex to $w$ on $\partial S(v)$, and the (combinatorial) distance from $w$ to $\sigma(w)$ is our parameter $s$. The map $w \mapsto \sigma(w)$ is clearly injective. This implies that the number $n_{r}^{+}$of positive excess vertices in $B\left(w_{0}, r\right)$ satisfies $n_{r+s}^{+} \leq n_{r}^{-}, r \in\{0,1,2, \ldots\}$. By choosing $s$ sufficiently large, we may therefore arrange to have the total excess in $B\left(w_{0}, r\right)$ to be less than $-\epsilon a^{r}$, for some $\epsilon>0$ and every $r \in\{0,1,2, \ldots\}$. It is clear that the number of vertices with zero excess in $B\left(w_{0}, r\right)$ is bounded by a constant (which may depend on $s$ ) times $n_{r}^{-}$. Hence, $\bar{E}_{m}<0$ for $\Gamma$.

By allowing $s$ to depend on the vertex in $T$, if necessary, we may arrange to have $\underline{E}_{m}=\bar{E}_{m}$; that is, $E_{m}$ exists, while maintaining $E_{m}<0$. We have thus demonstrated that the resulting surface is a counterexample in $F_{4}$ to the second implication in Nevanlinna's problem.

\section{A nonpositive CURVATURE EXAmple}

We now construct an example of a simply-connected, complete, parabolic surface $Y$ of nowhere positive curvature, with the property

$$
\int_{D(a, r)} \text { curvature }<-\epsilon \text { area }(D(a, r)),
$$

for some fixed $a \in Y$ and every $r>0$, where $D(a, r)$ denotes the open disc centered at $a$ of radius $r$, and $\epsilon>0$ is some fixed constant.

Consider the surface $\mathbb{C}=\mathbb{R}^{2}$ with the metric $|d z| / y$ in $P=\{z=x+i y: y \geq 1\}$, and $\exp (1-y)|d z|$ in $Q=\{y<1\}$. We denote this surface by $Y$. Let $\beta$ denote the curve $\{y=1\}$ in $Y$, i.e., the common boundary of $P$ and $Q$.

Let $Q^{\prime}$ denote the universal cover of $\{z \in \mathbb{C}:|z|>1\}$. Note that $Q$ is isometric to $Q^{\prime}$ via the map $z \mapsto \exp (i z+1)$. Hence, the curvature is zero on $Q$, and the geodesic curvature of $\partial Q$ is -1 . The geodesic curvature of $\partial P$ is 1 . Consequently, $Y$ has no concentrated curvature on $\beta$. The surface $Y$ is thus a "surface of bounded curvature", also known as an Aleksandrov surface (see [2], [7]). The curvature measure of $Y$ is absolutely continuous with respect to area; the curvature of $Y$ is -1 (times area measure) on $P$ and 0 on $Q$.

The surface $Y$ is parabolic, and the uniformizing map is the identity map onto $\mathbb{R}^{2}$ with the standard metric.

We will now prove (1) with $a=i$. Set $\beta_{r}=D(a, r) \cap \beta$. Note that the shortest path in $Y$ between any two points on $\beta$ is contained in $P$, and is the arc of a circle orthogonal to $\{y=0\}$. Using the Poincaré disc model, it is easy to see that there exists a constant $c>0$, such that

$$
c e^{r / 2} \leq \text { length } \beta_{r} \leq e^{r / 2} / c,
$$


where the right inequality holds for all $r$, and the left for all sufficiently large $r$. By considering the intersection of $D(a, r)$ with the strip $1<y<2$ it is clear that

$$
O(1) \operatorname{area}(P \cap D(a, r)) \geq \text { length } \beta_{r},
$$

for all sufficiently large $r$.

Consider some point $p \in Q$, and let $p^{\prime}$ be the point on $\beta$ closest to $p$. It follows easily (for example, by using the isometry of $Q$ and $Q^{\prime}$ ) that if $q$ is any point in $\beta$, then $d_{Q}(p, q)=d_{Q}\left(p, p^{\prime}\right)+d_{Q}\left(p^{\prime}, q\right)+O(1)$. Consequently, if $d(p, a) \leq r$, then there is an $s \in[0, r]$ such that $p^{\prime} \in \beta_{s}$ and $d_{Q}\left(p, p^{\prime}\right) \leq r-s+O(1)$. Furthermore, it is clear that the set of points $p$ in $Q$ such that $p^{\prime} \in \beta_{s}$ and $d_{Q}\left(p, p^{\prime}\right) \leq t$ has area $O\left(t^{2}+t\right) \times$ length $\beta_{s}$. Consequently,

$$
\operatorname{area}(Q \cap D(a, r)) \leq O(1) \sum_{j=0}^{r}(j+1)^{2} \text { length } \beta_{r-j} .
$$

Using (2), we have

$$
\operatorname{area}(Q \cap D(a, r)) \leq O(1) \text { length } \beta_{r},
$$

for all sufficiently large $r$.

Now, combining (3) and (4), we obtain (11) for all sufficiently large $r$. It therefore holds for all $r$.

\section{ACKNOWLEDGEMENTS}

The authors are grateful to B. Davis, D. Drasin, and especially to A. Eremenko for helpful discussions and their interest in this work.

\section{REFERENCES}

1. L. Ahlfors, Conformal invariants. Topics in geometric function theory, McGraw-Hill, Inc., 1973. MR 50:10211

2. A. D. Aleksandrov and V. A. Zalgaller, Intrinsic geometry of surfaces, Transl. Math. Monographs, Vol. 15, Amer. Math. Soc., Providence, RI, 1967. MR 35:7267

3. P. G. Doyle, Random walk on the Speiser graph of a Riemann surface, Bulletin Amer. Math. Soc., vol. 11, No. 2, pp. 371-377, 1984. MR 86b:58129

4. P. G. Doyle and J. L. Snell, Random walks and electric networks, Math. Assoc. of America, 1984. MR 89a:94023

5. R. Nevanlinna, Über die Riemannsche Fläche einer analytischen Funktion, Proceedings, International Congress of Mathematicians 1, Zurich, 1932.

6. R. Nevanlinna, Eindeutige analytische Funktionen, Springer-Verlag, 1936 (and also 1974). Translated as Analytic Functions, Die Grundlehren der mathematischen Wissenschaften, Band 162, Springer-Verlag, 1970. MR 43:5003

7. Yu. G. Reshetnyak, Two-dimensional manifolds of bounded curvature, In: Geometry IV. Encyclopedia of Mathematical Sciences, (Yu. G. Reshetnyak, ed.), Vol. 70, Springer-Verlag, pp. 3-163, 1993.

8. O. Lehto and K. I. Virtanen, Quasiconformal mappings in the plane, Second edition. SpringerVerlag, New York-Heidelberg, 1973. MR 49:9202

9. P. M. Soardi, Potential theory on infinite networks, Lecture Notes in Math., no. 1590, Springer-Verlag, New York, 1994. MR 96i:31005

10. S. Stoïlow, Leçons sur les Principes Topologiques de la Théorie des Fonctions Analytiques, Deuxième édition, augmentée de notes sur les fonctions analytiques at leurs surfaces de Riemann, Gauthier-Villars, Paris, 1956. MR 18:568b 
11. O. Teichmüller, Untersuchungen über Konforme und Quasikonforme Abbildung, Deutsch. Math. 3, pp. 621-678, 1938.

Department of Mathematics, Weizmann Institute of Science, Rehovot 76100, Israel

E-mail address: itai@math.weizmann.ac.il

Department of Mathematics, Purdue University, West Lafayette, Indiana 47907

E-mail address: smerenko@math.purdue.edu

Microsoft Research, One Microsoft Way, Redmond, Washington 98052

E-mail address: schramm@icrosoft.com 